\title{
ADAPTIVE MULTIMEDIA PACKET TRANSMISSION FOR BROADBAND IEEE 802.11 WIRELESS LANS
}

\author{
Joshua Wall, Jamil Khan \\ School of Electrical Engineering \& Computer Science \\ University of Newcastle, Australia \\ joshua.wall@studentmail.newcastle.edu.au,jamil.khan@newcastle.edu.au
}

\begin{abstract}
The IEEE 802.11 suite of WLAN standards are evolving to support an increase in emerging broadband multimedia applications, with their popularity attributed to simple, scalable and cost effective wireless broadband communication infrastructure. However, the most widely deployed generation of $802.11 \mathrm{x}$ based MAC protocols provide limited support for multimedia traffic, as no guarantee is provided on the quality of service $(\mathrm{QoS})$ that a particular type of traffic will receive. Furthermore, the legacy 802.11 MAC exhibits high protocol overhead due to control frames, channel access, and the probability of contention. This article presents an overview of MAC layer enhancements designed to provide QoS support and higher throughputs in next generation WLANs, including IEEE $802.11 \mathrm{e}$ and $802.11 \mathrm{n}$ standards. Comprehensive simulation results are provided using an OPNET simulation model.
\end{abstract}

\section{INTRODUCTION}

With the advancement of the widely deployed IEEE 802.11 WLAN standard [1], WLANs are being used to support an increasing demand in emerging broadband multimedia content and service delivery, including video conferencing, surveillance, high definition television (HDTV), voice over IP (VoIP), and entertainment services. In recent years, the 802.11 standard has evolved from being a simple low datarate network offering transmission rates of up to $11 \mathrm{Mb} / \mathrm{s}$ (802.11b), to a high data-rate ubiquitous network able to achieve a maximum transmission rate of $54 \mathrm{Mb} / \mathrm{s}(802.11 \mathrm{a} / \mathrm{g})$. Despite these advances, the legacy 802.11 MAC based on the carrier sense multiple access with collision avoidance (CSMA/CA) protocol, provides a 'best effort' delivery paradigm where the same transmission characteristics are used independent of the type of traffic being transmitted. As multimedia services are characterized by the requirement of strict throughput, delay, and delay variation (jitter) constraints, the provision of QoS mechanisms within the 802.11 protocol are vital for multimedia support. The recently ratified $802.11 \mathrm{e}$ amendment standard [2] defines enhancements to the MAC layer to enable QoS support. More recently, the 802.11n Task Group has been defining both MAC and Physical (PHY) layer enhancements, with a unified goal of achieving higher throughputs. A common objective for this increase is a throughput in excess of $100 \mathrm{Mb} / \mathrm{s}$, experienced at the MAC layer interface (MAC-SAP).

The legacy 802.11 MAC exhibits high protocol overhead due to contention, inter-frame spaces, and simple automatic repeat request (ARQ) schemes. Xiao and Rosdahl [3] have shown that the 802.11 MAC displays a theoretical maximum throughput limit, implying that a straightforward increase in
PHY bit-rate will not necessarily lead to a corresponding increase in MAC layer throughput. It is therefore necessary to develop MAC layer enhancements incorporating both QoS support and higher throughput provisioning, in order to facilitate the transmission of current and emerging broadband multimedia applications.

This article provides a brief overview of some of the mechanisms being considered for 802.11n to provide QoS support and higher throughputs at the MAC layer, including 802.11e integration, frame aggregation, and selective repeat ARQ schemes such as the 802.11e Block Acknowledgement mechanism. A simple novel adaptive packet transmission strategy is also presented, designed to provide higher throughput and reduced delay for multimedia traffic in both existing and future WLAN standards. The legacy 802.11 WLAN standard uses a simple stop and wait ARQ protocol that does not take into consideration channel conditions, or the requirements of the type of traffic being serviced, and therefore may exhibit inefficiencies due to channel access, contention periods, and the overhead associated with ACK transmission. The proposed adaptive packet transmission strategy employs a selective repeat ARQ algorithm, where the block size is dynamically changed to maximize throughput. The block size is defined as the total number of data packets a recipient can receive, after which a single collective acknowledgement packet must be returned.

This article is structured as follows: Section II briefly introduces the IEEE 802.11e ARQ scheme, and provides a brief overview of current higher throughput MAC enhancement mechanisms. Section III introduces an adaptive packet transmission strategy for multimedia traffic, based on the 802.11e Block ACK mechanism. An OPNET based simulation model is described in section IV, with simulation results and discussion presented in Section V. A brief conclusion is outlined in section VI.

\section{IEEE 802.11E/N MAC ENHANCEMENTS}

\section{A. 802.11e ARQ}

The 802.11e standard supports three different ARQ protocols as shown in Fig. 1, namely ACK (stop and wait), No ACK, and Block ACK (selective repeat). As mentioned previously, the legacy 802.11 WLAN standard typically only supports ACK mode, which is characterized by the transmission of a positive ACK by a recipient node to the originating node upon the successful reception of a data packet or segment, also know as a MAC Protocol Data Unit (MPDU). The acknowledgement of a MPDU occurs after a SIFS duration. If a MPDU is received in error or the transmission of the ACK is unsuccessful, the originating node retransmits the packet. If the packet has not been successfully transmitted after a 


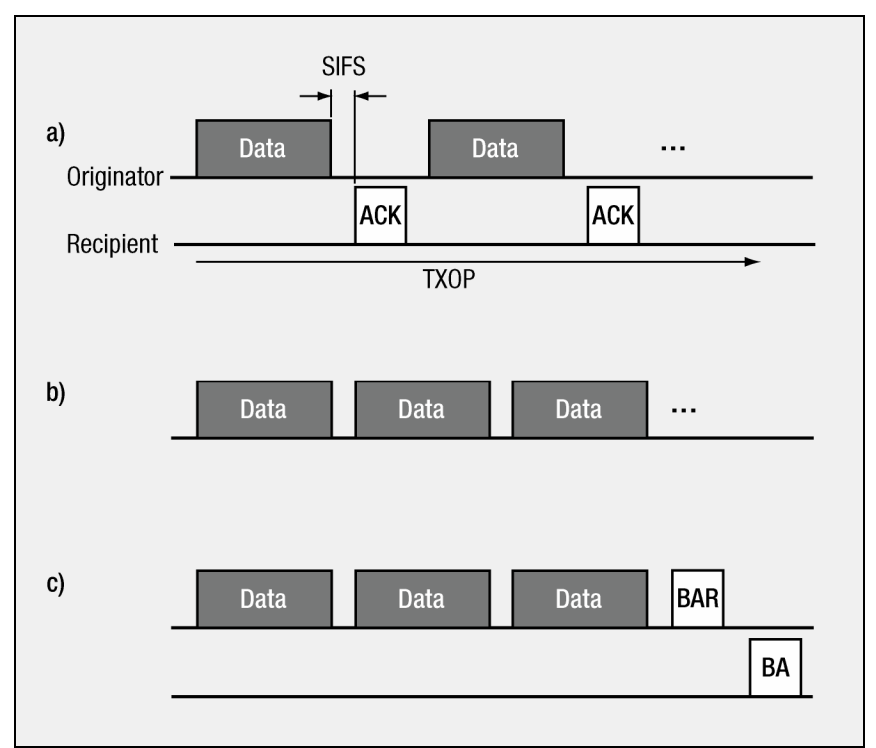

Figure 1: 802.11e ARQ - a) ACK, b) No ACK, c) Block ACK

predefined number of attempts, it is discarded. When operating in No ACK mode, a No Acknowledgement policy is used, where a data packet is transmitted once and only once regardless of whether it is received in error or not.

The Block ACK mechanism defined in the 802.11e allows a group of MPDUs to be transmitted consecutively within a Transmission Opportunity (TXOP), while bundling all corresponding acknowledgements into single collective ACK. A TXOP is defined as a bounded duration in which a node may perform packet bursting, where a sequence of SIFS separated packets are exchanged. Block ACK mode is initialized by an exchange of setup frames that negotiate parameters including buffer size and Block ACK type, where the Block ACK type is either immediate or delayed. After initialization, the originator may transmit a sequence of SIFS separated MPDUs, with the total number of MPDUs not exceeding the buffer size negotiated at the setup, or exceeding TXOP bounds. The originating node requests an ACK of outstanding MPDUs by sending a Block ACK Request (BAR) frame. If immediate Block ACK is used, the recipient responds by transmitting a Block ACK (BA) frame immediately after the BAR, which contains the acknowledgement state of data packets received from the originator. A BA frame can acknowledge up to 64 MPDUs, with each MPDU fragmented up to 16 times. Delayed Block ACK allows a node to acknowledge the BAR with an ACK, then prepare a BA response and send it in the earliest possible subsequent TXOP. The originator acknowledges the receipt of the BA with an ACK. This delayed approach is primarily intended to facilitate inexpensive implementations that use the processing power of a host, as well as to allow existing implementations to use this feature with minimal hardware changes.

Enhancements to the Block ACK mechanism currently being considered in $802.11 \mathrm{n}$ proposals [4] include the usage of implicit BAR when using frame aggregation. An aggregated frame need not include a BAR frame and the recipient interprets the receiving aggregation frame as though it includes a BAR frame. A compressed variant of the 802.11e
BA frame is also defined. The compression parameters are defined during the exchange of the Block ACK setup frames, based on whether to use fragmentation or not and the maximum number of MSDUs in the Block ACK window (block size). The size of the compressed BA frame is defined by the $\mathrm{BA}$ agreement, and is constant throughout the lifetime of the BA agreement.

The rules governing the adaptation of ARQ parameters and dynamic switching between the specified ARQ modes is out of the scope of the current 802.11e standard, and therefore provides an open and challenging research area. $\mathrm{Li}$ and van der Schaar [5] propose an adaptive QoS scheme for the transmission of layered video over an 802.11 WLAN network, through real-time adaptation of the ARQ Retry Limit parameter. Yuan et al. [6] present a high-performance MAC protocol called ADCA, which employs an adaptive block transmission concept within an infrastructure WLAN (centralized control using an Access Point) to reduce overhead and increase aggregate throughput. The proposed adaptive packet transmission strategy presented in this article is a distributed scheme, where the selective repeat ARQ block size is dynamically adapted based on feedback advertised by a recipient. Virtual ACK, No ACK and Block ACK modes are dynamically employed based on link state information.

\section{B. 802.11 In Frame Aggregation}

Although the 802.11e Block ACK mechanism offers increased throughput compared to 802.11, the overhead remains significant (almost 50 percent at $216 \mathrm{Mb} / \mathrm{s}$ PHY layer bit rate). Simoens et al. [7] show that increasing the number of MPDUs transmitted per TXOP from 16 to 64 does not bring significant improvement of MAC efficiency. This limitation comes from the fact that an SIFS of $16 \mu \mathrm{s}$ is inserted between each MPDU. To overcome this inefficiency, a technique known as frame aggregation is currently being studied within the 802.11n Task Group. Frame aggregation permits the encapsulation of multiple data packets or frames within one aggregated super frame, so as to eliminate the need for multiple sets of overhead associated with separate frame transmission. An aggregated frame can contain frames or fragments of frames, which are addressed to a common recipient. The number of frames that are permitted to be aggregated is limited by restraints such as the ARQ window or recipient buffer size.

Abraham et al. [8] present an overview of 802.11n high throughput enhancements, including a MAC layer frame aggregation concept. Xiao [9] proposes implementation specific frame aggregation techniques, namely concatenation and packing. Concatenation, as illustrated in Fig. 2a, involves the aggregation of multiple PHY layer Protocol Data Units (PPDU) into one single PHY layer Service Data Unit (PSDU). A concatenated frame includes a concatenation header $(\mathrm{CH})$, which identifies itself as a concatenated frame type, as well as indicating the number of PPDUs succeeding it and the total aggregated length. When a recipient receives a concatenated frame, it decomposes it into separate frames by using preamble and Frame Check Sequence (FCS) patterns to determine the individual PPDU boundaries. If all PPDUs are received correctly, the concatenated frame is acknowledged 
according to its ARQ policy. Packing, as shown in Fig. 2b, is defined as the act of combining multiple MSDUs or higher layer fragments into a single PSDU. Packing is similar to concatenation, with the difference that the aggregated frames for the packing mechanism form one PSDU, where as concatenation forms multiple PPDUs within a single PSDU. More simply, packing involves combining multiple frames into one larger frame with only one MAC header, called the Packing Header ( $\mathrm{PH})$, and one FCS. It is because of this that an aggregated frame using the packing technique must contain a length field preceding each MSDU or payload. The packing technique is more efficient then concatenation, but requires greater processing time due to the combing and decomposing of frames.

\section{ADAPTIVE PACKET TRANSMISSION}

Section II described the different ARQ modes defined in the 802.11e standard. As mentioned earlier, ACK mode may not be the most efficient type of ARQ to use for a particular class of traffic, as the transmission of an ACK packet and its associated SIFS consumes channel bandwidth and introduces additional delay. Equations (1), (2) and (3) show the minimum time required for the transmission of 64 MPDUs within a single TXOP using ACK, No ACK, and Block ACK mode respectively, assuming that there is only one transmitting node that always has a data packet to send. All numerical values and the meanings of the terms used in (1), (2) and (3) are explicitly detailed in Table 1.

Using the parameters from Table 1, the minimum time to transmit a block of 64 MPDUs in ACK mode is equal to $4.94 \mathrm{~ms}$, with No ACK and Block ACK modes taking $3.55 \mathrm{~ms}$ and $3.63 \mathrm{~ms}$ respectively. This shows that Block ACK mode for the specified scenario is almost 30\% faster than ACK mode, and only $2 \%$ slower than No ACK mode.

Despite this reduction in transmission time, the retransmission process in ACK and Block ACK modes provide a higher probability of successful transmission, which data services or links experiencing poor channel conditions may require. To compromise these conflicting requirements, we propose a simple adaptive packet transmission strategy based on the 802.11e Block ACK mechanism. The proposed algorithm dynamically adjusts the block size $\mathrm{B}_{\mathrm{s}}$ depending on both channel conditions and application requirements. For Block ACK mode, $B_{s}=\{2,3, \ldots, 64\}$. Moreover, virtual ACK and No ACK modes are invoked by setting $B_{s}=1$ for $A C K$

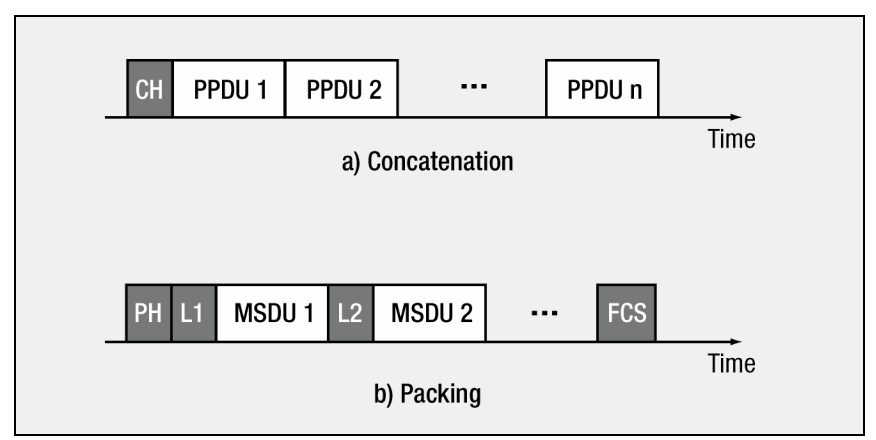

Figure 2: Frame Aggregation techniques

$$
\begin{gathered}
T_{A C K}=\left(t_{M P D U}+2 t_{p}+2 t_{S I F S}+t_{A C K}\right) * n_{p k t s}+t_{D I F S} \\
T_{\text {NoACK }}=\left(t_{M P D U}+t_{p}+t_{S I F S}\right) * n_{p k t s}+t_{D I F S} \\
T_{\text {BlockACK }}=\left(t_{M P D U}+t_{p}+t_{S I F S}\right) * n_{p k t s} \\
+t_{D I F S}+t_{B A R}+t_{S I F S}+t_{B A}+2 t_{p}
\end{gathered}
$$

\begin{tabular}{|c|c|c|}
\hline Parameter & Value & Comment \\
\hline$t_{p}$ & $1 \mu \mathrm{s}$ & Propagation delay $(300 \mathrm{~m})$ \\
\hline$t_{M P D U}$ & $37.9 \mu \mathrm{s}$ & $\begin{array}{l}\text { MPDU transmission time }(\text { Size }= \\
256 \text { Bytes, Data rate }=54 \mathrm{Mb} / \mathrm{s})\end{array}$ \\
\hline$t_{\text {SIFS }}$ & $16 \mu \mathrm{s}$ & $\begin{array}{l}\text { Duration of Short Inter-Frame } \\
\text { Space (SIFS - 802.11a) }\end{array}$ \\
\hline$t_{D I F S}$ & $34 \mu \mathrm{s}$ & $\begin{array}{l}\text { Duration of Distributed Inter- } \\
\text { Frame Space (DIFS - 802.11a) }\end{array}$ \\
\hline$t_{A C K}$ & $4.6 \mu \mathrm{s}$ & $\begin{array}{l}\text { ACK transmission time } \\
(\text { Data rate }=24 \mathrm{Mb} / \mathrm{s})\end{array}$ \\
\hline$t_{B A R}$ & $8 \mu \mathrm{s}$ & $\begin{array}{l}\text { Block ACK Request transmission } \\
\text { time }(\text { Data rate }=24 \mathrm{Mb} / \mathrm{s})\end{array}$ \\
\hline$t_{B A}$ & $50.7 \mu \mathrm{s}$ & $\begin{array}{l}\text { Block ACK transmission time } \\
(\text { Data rate }=24 \mathrm{Mb} / \mathrm{s})\end{array}$ \\
\hline$n_{p k t s}$ & 64 & Block size (packets) \\
\hline
\end{tabular}

Table 1: Transmission time parameters for 802.11a

mode, and $\mathrm{B}_{\mathrm{s}}=\infty$ for No ACK mode.

Although the No Acknowledgement policy is the most efficient in regards to protocol overhead, there is a trade-off in reliability, as data packets are transmitted only once regardless of their error status. As multimedia services along with other periodical or interactive traffic classes can often cope with a small loss rate through techniques such as error concealment or Forward Error Correction (FEC), the use of No ACK mode can maximize performance.

The adaptive transmission algorithm dynamically adapts the ARQ block size based on channel conditions, in order to optimize throughput. The channel quality is obtained using a distributed receiver initiated scheme, where a recipient maintains channel state information, and periodically makes an intelligent decision as to whether to advise the originating node of this information. If the channel state is deemed to have changed beyond a certain threshold, determined from link layer state information such as bit-error rate (BER), the recipient sends a custom control packet informing the originator of this value. An alternative solution to this currently being researched, is the use of a closed-loop feedback system, such as receiver assisted link adaptation (LA) schemes proposed for 802.11n [4]. Under these schemes, closed-loop modulation and coding scheme (MCS) adaptation is provided by the exchange of MRQ (MCS Request) and MFB (MCS Feedback) elements carried in an enhanced version of the 802.11 RTS/CTS control frames. A node receiving an MFB uses the information contained in it to adapt its transmission parameters. This information could also be used by the adaptive packet transmission algorithm to assist in ARQ block size adaptation. Fig. 3 shows the adaptive packet transmission algorithm signalling sequence. 


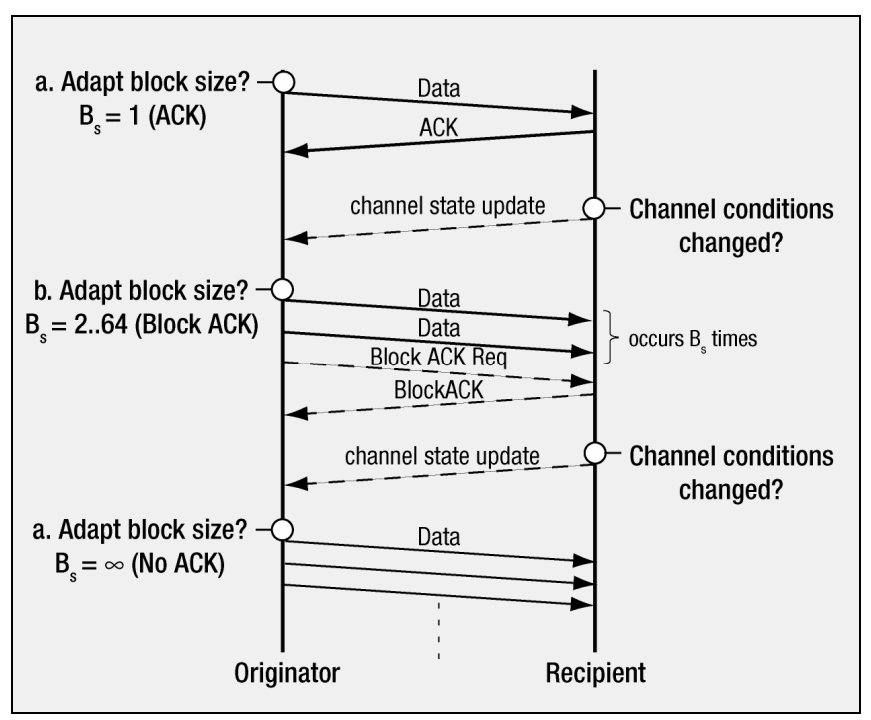

Figure 3: Adaptive packet transmission signalling sequence

If the channel state reflects a need to change the block size, the recipient node informs the originating node of this value using a custom control frame. The custom control frames are sent using a No Acknowledgement policy, as the short periodic nature of the channel state calculation provides high probability that the originating node will be signalled, regardless of unsuccessful transmissions. Upon receiving channel state information, the originating node performs block size adaptation so as to maximize throughput whilst implicitly maintaining application QoS requirements through the 802.11e EDCA mechanism.

The proposed adaptive transmission strategy is coupled with the 802.11e mechanism of discarding MSDUs from the transmitter queue based on an AC-specific delay threshold called a MSDU lifetime. A timer is started when a MSDU is first passed to the MAC layer, and if it exceeds its associated MSDU lifetime before successful transmission, all remaining fragments of that MSDU are discarded by the originating node without any further attempt to complete delivery. This is advantageous for multimedia traffic, as if a multimedia data packet is not transmitted within a certain delay, it often exceeds its effective usefulness or ability to contribute at the receiving application, therefore consuming unnecessary bandwidth. Unequal error protection for multimedia traffic could also be supported by allowing variations in the ARQ policy when operating in the Block ACK or No ACK modes. High priority packets, such as video key frames or scalable video base layers, can use an acknowledgement policy to ensure a higher transmission probability with smaller delay.

\section{Simulation}

\section{A. OPNET model}

A comprehensive discrete event simulation model using OPNET is used to analyze the performance of the proposed adaptive packet transmission strategy operating in conjunction with 802.11e EDCA mechanism. The model uses the 802.11a PHY layer, which supports PHY layer data rates of up to $54 \mathrm{Mb} / \mathrm{s}$. The $5 \mathrm{GHz}$ wireless channel is simulated in OPNET using a log-distance path loss model of exponent 3.6, and a shadowing variance equal to $5 \mathrm{~dB}$ with typical NLOS channel characteristics. The link adaptation is based on the knowledge of the Signal-to-Noise Ratio (SNR), which is assumed to be perfectly known. All stations transmit at a constant power equal to $200 \mathrm{~mW}$. The SNR for each data packet is mapped to a BER value through the use of empirical OPNET modulation curves. To further increase the accuracy of the channel model, separate modulation curves are used for each of the 8 modulation and coding schemes used in 802.11a. The adaptive packet transmission strategy is simulated by using a PER metric as the switching threshold, where by the block size is dynamically adapted between the values of $B_{s}=1$ and $B_{s}=\infty$ depending on the PER. A 5\% PER threshold is used, with the PER calculated from a historical time window of 1 second duration. A receiver error correction threshold value of $10^{-3}$ is assumed.

\section{B. Simulation Scenario}

The adaptive packet transmission algorithm is simulated using three different scenarios as follows: Scenario 1 consists of two stations in a cell, where at any given time there is only one active station that always has a packet to send, with the other station performing reception and acknowledgment tasks. The amount of traffic generated ensures that the system operates in saturation conditions when transmitting at the highest possible bit rate. A packet size of 1500 bytes is used. Scenario 2 is used to analyze the use of the Adaptive ARQ algorithm in a multi-station environment. It has similar traffic generation characteristics to scenario 1 . Scenario 3 consists of 5 stations generating multimedia traffic using a MPEG-4 video trace [10]. An additional station transmitting background data traffic is used to ensure that the system operates in saturation conditions. The multimedia stations access the channel through a higher priority AC.

\section{RESUlts}

Fig. 4a shows the throughput performance against increasing SNR for a single transmitting station using both the legacy 802.11 standard and the adaptive ARQ algorithm. It can be seen that the adaptive ARQ strategy provides throughput gains during periods when the channel conditions are good enough to allow Block ACK or No ACK modes to be invoked. A maximum throughput gain of $10 \mathrm{Mb} / \mathrm{s}$ is achieved for SNR values greater than $24 \mathrm{~dB}$, whilst smaller gains occur for all SNR values above 4dB. Fig. $4 \mathrm{~b}$ shows the effect of increasing the number of transmitting stations within a basic service set (BSS). A $3 \mathrm{Mb} / \mathrm{s}$ throughput gain over the legacy 802.11 standard is achieved over the entire spectrum of 2 to 50 transmitting stations when Adaptive ARQ is employed, whilst the concurrent use of Adaptive ARQ and TXOPs provide gains of up to $10 \mathrm{Mb} / \mathrm{s}$. Fig. 5 shows the aggregate throughput, delay, and PER results when transmitting MPEG4 video data using the adaptive ARQ strategy. Whilst the Adaptive ARQ algorithm achieves incremental gains in this scenario for both throughput and delay, the main advantage can be seen from Fig. 5c, where the PER for a single MPEG-4 stream is reduced by a factor of almost $50 \%$. This is extremely beneficial when it comes to the transmission of 


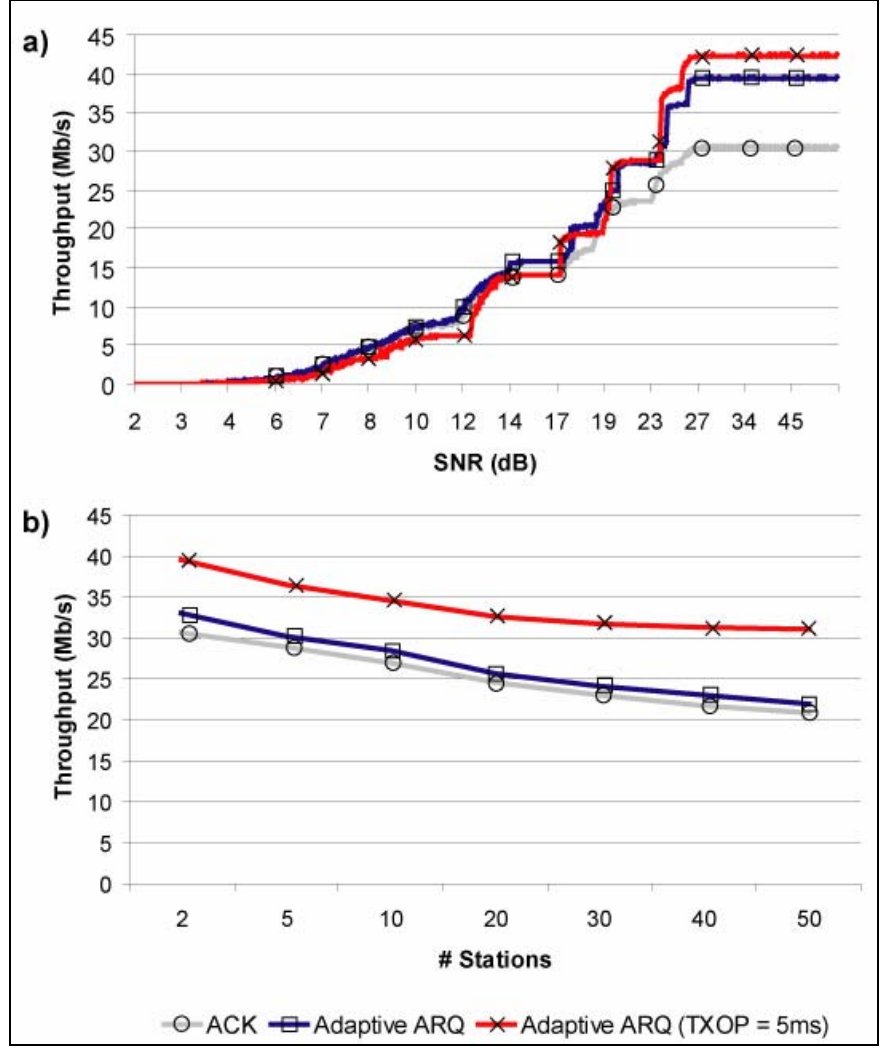

Figure 4: Throughput - a) Single station, b) Multi-station video, as the subjective quality of video can often be severely affected by packet loss, which then can only be alleviated by various techniques such as error concealment. It is shown that further improvements are achieved in all scenarios when frame bursting techniques (802.11e TXOP) are used in conjunction with the adaptive packet transmission algorithm.

\section{CONCLUSION}

This article introduces several IEEE 802.11e/802.11n MAC layer enhancement techniques, which can be employed to improve the throughput and reliability of a broadband WLAN for multimedia traffic. Legacy MAC layer architecture is unable to efficiently utilize the offered PHY layer data rate because of high MAC layer overhead. Some of the MAC layer overhead is introduced by ARQ procedures used to support different classes of traffic, irrespective of their QoS requirements. In this paper we proposed an adaptive block ARQ technique and demonstrated that it could significantly improve the throughput and QoS of a broadband WLAN under multiple scenarios. We conclude that to improve QoS the TXOP mechanism combined with the Block ACK or frame aggregation techniques can offer very high throughput and minimum packet transmission delay. Simulation results show that the adaptive ARQ technique when combined with packet bursting offers significant advantages in terms of packet loss for video traffic. This article has also highlighted that an enhanced 802.11 MAC layer providing QoS support and higher throughputs, is both necessary and important to support the diverse range of emerging broadband multimedia applications in current and next generation WLANs.

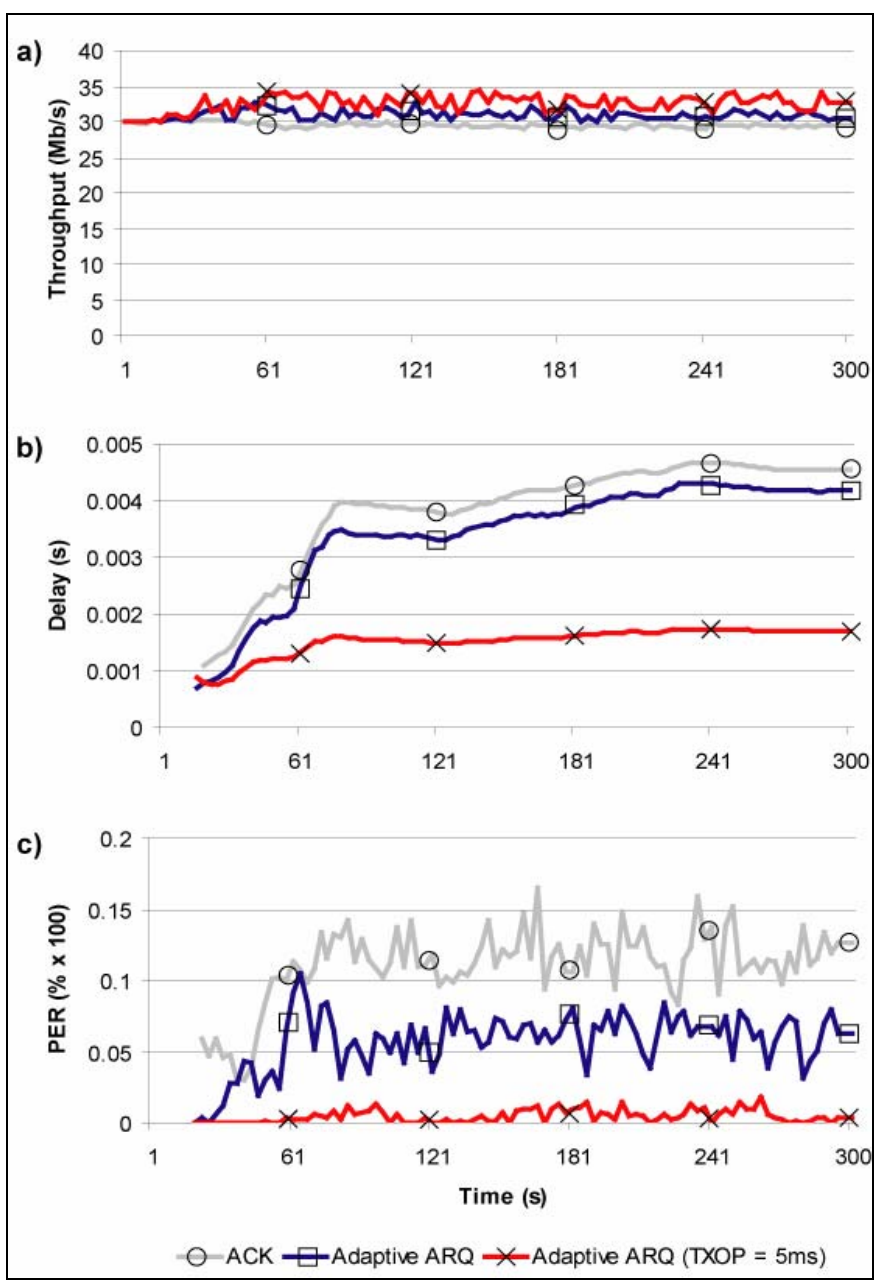

Figure 5: MPEG-4 video: a) Throughput, b) Delay, c) PER

\section{REFERENCES}

[1] IEEE Std 802.11, 1999 Edition, Part 11: Wireless LAN Medium Access Control (MAC) and Physical Layer (PHY) Specifications.

[2] IEEE Std 802.11e -2005, Amendment to IEEE Std 802.11, 1999 Edition (Reaff 2003), Amendment 8: Medium Access Control (MAC) Quality of Service Enhancements.

[3] Y. Xiao and J. Rosdahl, "Throughput and Delay Limits of IEEE 802.11," IEEE Commun. Lett., vol. 6, no. 8, Aug. 2002, Pages 355-57.

[4] Enhanced Wireless Consortium, "HT MAC Specification", Ver. V1.24.

[5] Q. Li, M. van der Schaar, "Providing Adaptive QoS to Layered Video Over Wireless Local Area Networks Through Real-ime Retry Limit Adaptation", IEEE Trans. on Multimedia, Vol. 6, No. 2, April 2004

[6] Y. Yuan, G. Daqing, W. Arbaugh, Z. Jinyun,"High-performance MAC for high-capacity wireless LANs", ICCCN 2004, Pages 167 - 172.

[7] S. Simoens, P. Pellati, J. Gosteau, K. Gosse, C. Ware, "The evolution of $5 \mathrm{GHz}$ WLAN toward higher throughputs", IEEE Wireless Communications, Vol.10, Issue 6, Dec. 2003, Pages 6-13.

[8] S. Abraham, A. Meylan, S.Nanda, "802.11n MAC Design and System Performance", ICC 2005.

[9] Yang Xiao, "Efficient MAC Strategies for the IEEE 802.11n wireless LANs", Wireless Comms. \& Mobile Comp. 2006, 6:453-466, Wiley InterScience.

[10] F.H.R.Fitzek, M. Zorzi, P. Seeling, M. Reisslein, "Video and audio trace files of pre-encoded video content for network performance measurements", CCNC 2004, Pages 245 - 250. 